\title{
BMJ Open Individual, social and environmental determinants of sleep among women: protocol for a systematic review and meta-analysis
}

\author{
Lydi-Anne Vézina-Im, Jennette P Moreno, Debbe Thompson, Theresa A Nicklas, \\ Tom Baranowski
}

To cite: Vézina-Im L-A, Moreno JP, Thompson D, et al. Individual, social and environmental determinants of sleep among women: protocol for a systematic review and meta-analysis. BMJ Open 2017;7:e016592. doi:10.1136/ bmjopen-2017-016592

- Prepublication history for this paper is available online. To view these files please visit the journal online (http://dx.doi. org/10.1136/bmjopen-2017016592).

Received 24 February 2017

Revised 5 May 2017

Accepted 9 May 2017

CrossMark

Department of PediatricsNutrition, Children's Nutrition Research Centre, Baylor College of Medicine, Houston, Texas, USA

Correspondence to Dr Lydi-Anne Vézina-Im; lydianne.vezina-im@bcm.edu

\section{ABSTRACT}

Introduction Sleep is important to promote optimal health and avoid negative health outcomes. Short-duration and low-quality sleep may be more common and more detrimental among women compared with men. Identifying the determinants of behaviour is one of the first steps in designing effective interventions. To our knowledge, no systematic review has identified the individual, social and environmental determinants of sleep among adult women. Methods and analysis Studies reporting data on adult women from 18 to 64 years of age will be included. On the basis of ecological models of health behaviour and sleep, the types of determinants that will be included in the review are individual (eg, demographic, psychological and behavioural), social (eg, family) and environmental (eg, physical environment and policies) determinants. Observational (cross-sectional and longitudinal) and experimental studies will be included. MEDLINE/PubMed, PsycINFO, CINAHL, EMBASE and Proquest Dissertations and Theses will be investigated. Data will be extracted independently by two reviewers using a standardised data extraction form. The quality of observational studies will be assessed using the National Institute of Health Quality Assessment Tool for Observational Cohort and CrossSectional Studies and the quality of experimental studies will be assessed using the Effective Public Health Practice Project Quality Assessment Tool for Quantitative Study. If there is a sufficient number of studies reporting data on a similar determinant among a similar population $(k>5)$, a meta-analysis of the results will be performed with a random-effects model. If between-study heterogeneity is high $\left(1^{2} \geq 75 \%\right)$, it will be investigated through sensitivity analyses and meta-regression.

Ethics and dissemination Formal ethical approval is not required as no primary data will be collected. The results will be published in a peer-reviewed journal. This review will provide valuable information to those interested in developing empirically based sleep interventions among women. PROSPERO registration number CRD42017056894.

\section{INTRODUCTION}

Sleep is important to promote optimal health and avoid negative health outcomes. When investigating sleep's association with health, two aspects are the most important:

\section{Strengths and limitations of this study}

- To our knowledge, no previous review has identified the individual, social and environmental determinants of sleep among women.

- Investigating simultaneously the individual, social and environmental determinants of women's sleep will allow us to ascertain which determinants contribute the most to adequate sleep in women and provide a more comprehensive picture of factors that affect women's sleep.

- This review will also provide information on whether the determinants of sleep differ according to age (eg, childbearing-age vs older women), pregnancy status (pregnant or not) and ethnicity to inform the development of interventions to promote maternal, foetal and child health in various populations.

- Since the investigation of determinants of sleep is a rather new field, the number of studies to include in the review might be low.

- The low number of studies and significant heterogeneity between studies might preclude performing meta-analyses of the results.

duration and quality of sleep. ${ }^{1}$ A recent systematic review/meta-analysis indicated that short sleep was significantly associated with obesity (risk ratio $(\mathrm{RR})=1.38$; $95 \%$ confidence interval (CI) 1.25 to 1.53 ), diabetes mellitus ( $\mathrm{RR}=1.37$; 95\% CI 1.22 to 1.53 ), coronary heart diseases $(\mathrm{RR}=1.26 ; 95 \% \mathrm{CI}$ 1.15 to 1.38$)$, hypertension ( $\mathrm{RR}=1.17$; $95 \%$ CI 1.09 to 1.26 ), cardiovascular diseases $(\mathrm{RR}=1.16 ; 95 \%$ CI 1.10 to 1.23$)$ and mortality $(\mathrm{RR}=1.12 ; 95 \%$ CI 1.08 to 1.16$) .{ }^{2}$ Two recent systematic reviews/meta-analyses indicated that poor sleep quality was associated with increased haemoglobin A1c (weighted mean difference: $0.35 \%$; $95 \%$ CI 0.12 to 0.58$)^{3}$ in adults diagnosed with type 2 diabetes (T2D) and with overweight/obesity in young people (ie, children, adolescents and young adults) (OR=1.46; $95 \%$ CI 1.24 to 1.72$){ }^{4}$ 
Few studies have assessed whether gender modifies the association between sleep and health outcomes in adults. In a study with a 10-year follow-up, non-pregnant women under 40 years of age whose sleep duration decreased from adequate to short were at risk for weight gain $(\mathrm{OR}=1.85$; $95 \% \mathrm{CI} 1.14$ to 3.02$)$ and higher waist circumference $(\mathrm{OR}=1.84 ; 95 \% \mathrm{CI} 1.20$ to 2.81$){ }^{5} \mathrm{~A}$ recent meta-regression indicated that short sleep duration ( $<7$ hours/night) was significantly associated with all-cause mortality in women, but not in men. ${ }^{6}$ National data from the USA revealed women were more likely than men to frequently have trouble falling and staying asleep and to wake up feeling unrested, ${ }^{7}$ and that being female was associated with insufficient sleep. ${ }^{8}$ Among patients with T2D, female gender $(\mathrm{OR}=1.72)$ was one of the strongest predictors of any sleep symptom (eg, difficulty falling asleep, daytime sleepiness and difficulty staying awake). ${ }^{9}$ Hormonal factors, such as menstrual cycle, pregnancy and menopause, could contribute to poor sleep quality among women. ${ }^{10} 11$ This all suggests that short-duration and low-quality sleep may be more common and more detrimental among women of all ages. Another demographic variable associated with sleep is ethnicity, with recent reviews reporting that inadequate sleep duration and poorer sleep were more prevalent among low-income and ethnic minorities in the USA. ${ }^{12} 13$

Pregnant women and those of childbearing age (18 to 44 years) are an important target for interventions aimed at improving sleep since their behaviour can impact their own and their child's health. ${ }^{14}$ Among pregnant women, short sleep duration during pregnancy was linked to increased risk for gestational hyperglycaemia ${ }^{15}$ and gestational diabetes mellitus. ${ }^{16}$ Maternal short sleep duration in the first year postpartum was associated with higher adiposity at 3 years postpartum. ${ }^{17}$ National data from the USA revealed that diagnosed sleep disorders, report of having trouble sleeping and very short sleep ( $\leq 5$ hours/ night) or short sleep (6 hours/night) were more common among childbearing-age women compared with pregnant women. ${ }^{18}$ These data suggest the need to design interventions to promote sleep among pregnant and childbearing age women.

Intervention Mapping ${ }^{19}$ and the Behaviour Change Wheel $^{20}$ are comprehensive, empirically based approaches to designing health promotion interventions. Both recommend identifying the determinants of behaviour to understand what needs to be changed (ie, intervention targets) as the first step in developing an effective health promotion intervention. Ecological models of health behaviour can be used to guide the search for determinants. Ecological models recognise multiple levels of influence (or determinants) on specific health behaviours, such as intrapersonal (biological, psychological), interpersonal (social, cultural), organisational, community, physical environment and public policy. ${ }^{21}$ Interventions based on ecological models of health behaviour should target specific behaviours and their multiple levels of influence. ${ }^{21}$ Recently, socioecological models of determinants of sleep among adults have been proposed. In an initial review, the following terminology was employed to classify influences on sleep in adults: microsystem (eg, demographics), mesosystem (eg, family) and macrosystem (eg, physical environment) ${ }^{1}$ In a subsequent review, the same author used the following terms to define the levels of influence on sleep in adults: individual (eg, genetics), social (eg, home) and societal levels (eg, public policy) ${ }^{13}$ In another review, determinants of sleep in adults were classified as proximal factors (eg, demographics), intermediate factors (eg, social relationships) and distal factors (eg, social conditions and policies). ${ }^{12}$ Although different terms were used to classify the determinants of sleep in adults, a consensus appears to have formed around three levels of influence on sleep: individual (eg, demographic, psychological and behavioural), social (eg, family) and environmental (eg, physical environment and policies) determinants.

A previous review on the social and environmental determinants of sleep among children, adolescents and adults identified living in a disadvantaged neighbourhood, screen use, bedroom environment and family factors, such as maternal depression, marital status, family conflict and parental financial problems, as factors that can contribute to poor sleep in those populations. ${ }^{22}$ This review, however, did not look at the behavioural determinants of sleep (eg, caffeine consumption), did not distinguish between sleep duration and quality, and did not focus specifically on women. Finally, the little evidence available on the topic of gender differences in the determinants of sleep indicates that they vary between men and women, ${ }^{23} 24$ suggesting the need to investigate these sexes separately.

In sum, short and poor sleep can be associated with diverse negative health conditions, especially among women. Before interventions can be successfully designed or implemented, the determinants of sleep in this specific population must be identified. To our knowledge, no previous review has identified the individual, social and environmental determinants of sleep among women; verified if the determinants are different in pregnant and childbearing age women compared with other women; or examined ethnic differences among women. The present systematic review will answer the following questions:

- What are the individual, social and environmental determinants of sleep duration and sleep quality in women?

- Which determinants are most strongly related to adequate sleep in women?

- Do the determinants of sleep vary according to demographic characteristics, such as age (eg, childbearing-age vs older women), pregnancy status (pregnant or not) or ethnicity?

\section{METHODS AND ANALYSIS}

The study protocol was registered in PROSPERO (www. crd.york.ac.uk/PROSPERO/) in February 2016 (no. 
CRD42017056894). This protocol follows the Preferred Reporting Items for Systematic Review and Meta-Analysis Protocols (PRISMA-P) checklist $^{25}$ (see online supplementary file 1 for the completed checklist). Any amendments to the protocol will be tracked and dated in PROSPERO.

\section{Study eligibility criteria \\ Population}

Only studies reporting data on adult women from 18 to 64 years of age will be included. This age range was selected given that the sleep duration recommendation from the US National Sleep Foundation is the same across this age range: from 7 to 9 hours per night. ${ }^{26}$ Studies on special populations, such as pregnant women, and women with health problems (eg, breast cancer) or sleep disorders (eg, apnoea) will also be included, but they will be treated separately in the analyses. If a study reports data on men and women separately, only the data on women will be included and analysed. In instances where the determinants of sleep in adults are not separated by gender, the authors of the articles will be personally contacted to verify whether they can provide the data on determinants pertaining only to women.

\section{Determinants}

Determinants are 'factors thought to be associated with an outcome of interest'. ${ }^{27}$ While cross-sectional studies cannot establish directionality of association or causality between a determinant and the outcome of interest, ${ }^{28}$ they may identify possible determinants, and thereby will be included in this review, but treated separately in the analyses. The types of determinants that will be included are individual (eg, demographic, such as age, pregnancy status and ethnicity; psychological, such as stress and depression; and behavioural, such as caffeine consumption), social (eg, family, such as marital conflict and social support) and environmental (eg, physical environment, such as the bedroom environment and neighbourhood characteristics; and policies, such as offices with flexible work hours policies) determinants (see figure 1). This list of individual, social and environmental determinants of sleep among women is not exhaustive. Additional determinants of sleep will be included and classified at one of those three levels when we conduct the systematic review. This classification of determinants is based on ecological models of health behaviour ${ }^{21}$ and recent socioecological models of determinants of sleep in adults. ${ }^{1213}$

\section{Outcome}

The outcomes will be sleep duration (in minutes or hours), either self-reported or measured objectively (eg, by wrist actigraphy or total sleep time by polysomnography), and sleep quality, either self-reported (eg, Pittsburgh Sleep Quality Index ${ }^{29}$ ) or measured objectively (eg, number of awakenings at night measured by wrist actigraphy or wakefulness after sleep onset, rapid eye movement and stage sleep by polysomnography).

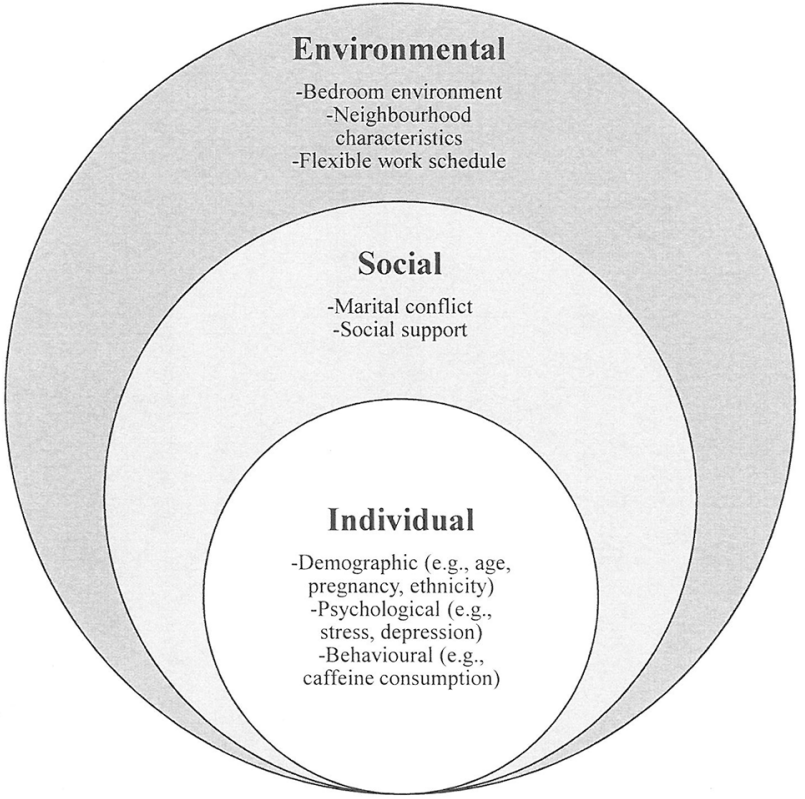

Figure 1 Ecological model of determinants of sleep among women.

\section{Study designs}

Observational epidemiological studies-either crosssectional (eg, surveys) or longitudinal (eg, cohort studies) - and experimental (quasi-experimental and randomised controlled trials) studies that assess the association among individual, social and environmental variables with sleep will be included.

\section{Search strategy}

The following databases will be investigated: MEDLINE/ PubMed (1946+), PsycINFO (1887+), CINAHL (1937+) and EMBASE (1980+). Proquest Dissertations and Theses (1861+) will also be investigated for unpublished trials (a form of grey literature). There will be no restriction on the year nor the country of publication of the articles. In each database, the search strategy will include terms related to two major themes: sleep and women (see table 1 for the complete search strategy for MEDLINE/PubMed). No term related to determinants will be included as a preliminary search revealed that it was too restrictive. Other terms like correlates and predictors were too broad. Only studies written in English or French will be included. The results of the search strategy will be reported in a PRISMA flow chart. ${ }^{30}$

\section{Study selection and data extraction}

First, all the articles retrieved from the search strategy will be screened for possible duplicates. Second, clearly irrelevant articles will be excluded according to their title and abstract. The remaining articles will be fully retrieved (full text) and two authors will independently assess them for eligibility. We will also verify if some studies report the same results based on the same sample. In those instances, we will only select the study with the best methodological qualities (eg, longitudinal vs cross-sectional 
Table 1 Complete search strategy for MEDLINE/PubMed

\begin{tabular}{|c|c|}
\hline Name of the database (range of dates) & MEDLINE/PubMed (1950+) \\
\hline \multicolumn{2}{|l|}{ Dates of the search } \\
\hline Initials of the person who will run the search & LAVI \\
\hline
\end{tabular}

study) and that reports the most information (eg, all the determinants vs a selection of determinants of sleep or univariate vs multivariate analyses) to avoid duplication of results and attributing more weight to these studies in the meta-analysis of the results.

Data will be extracted independently by two reviewers using a standardised data extraction form that will previously be pilot tested with five randomly selected articles. The quality of observational epidemiological studies will be assessed using the National Institute of Health (NIH) Quality Assessment Tool for Observational Cohort and Cross-Sectional Studies. ${ }^{31}$ The quality of experimental studies will be assessed using the Effective Public Health Practice Project (EPHPP) Quality Assessment Tool for Quantitative Study. ${ }^{32}$ This tool is recommended by the Cochrane Collaboration ${ }^{33}$ and can be used for different kinds of quantitative study designs (eg, randomised controlled trials and quasi-experimental studies). Types of determinants of sleep will be classified as individual, social or environmental determinants based on ecological models of health behaviour ${ }^{21}$ and socioecological models of determinants of sleep in adults. ${ }^{1213}$ Disagreements at each step will be resolved by discussion. When no consensus is reached, a third reviewer will resolve the discrepancy.

\section{Data synthesis and analyses}

The results of the studies included in the systematic review will be reported descriptively in a summary table, which will present information on the objective of the study, the population targeted, the study design and the quality of the study according to the NIH or the EPHPP tool, the characteristics of the sample, the behavioural measure, the covariates used in the statistical analyses and the results on sleep duration and/or quality. The results on sleep duration and/or quality will be reported in effect sizes, such as OR for dichotomous outcomes (eg, short vs adequate sleep) or standardised mean differences (SMD) for continuous outcomes (eg, sleep duration in hours). All effect sizes will be zero order (ie, no covariates will be included in the computation of the effect size). The ORs will be converted to Cohen's $d^{34}$ to facilitate interpretation and allow comparison with other SMD and standard effect sizes reported in other studies. A Cohen's d of 0.20 is considered a small effect size, 0.50 a medium effect size and 0.80 a large effect size. ${ }^{34}$

If we have a sufficient number of studies reporting data on a similar determinant among a similar population $(\mathrm{k}>5),{ }^{35}$ a meta-analysis of the results will be performed with a random-effects model. Random effects will be used because we expect the magnitude of the effect sizes will vary across studies given the differences in samples and determinants across studies. ${ }^{36}$ Each study will be weighted according to its sample size when we compute the pooled effect sizes. Between-study heterogeneity will be verified using Cochran's $\mathrm{Q}^{37}$ and the $\mathrm{I}^{2}$ statistic $^{38}$ as measures of the percentage of total variation in estimated effects that is due to heterogeneity rather than chance. ${ }^{39}$ A significant $Q$ statistic $(\mathrm{p}<0.05)$ indicates significant heterogeneity between the studies while an $\mathrm{I}^{2}$ statistic of $25 \%$ is considered low heterogeneity, $50 \%$ moderate heterogeneity and $75 \%$ high heterogeneity. ${ }^{40}$ If between-study heterogeneity is high $\left(\mathrm{I}^{2} \geq 75 \%\right)$, it will be investigated through sensitivity analyses and meta-regression. Publication bias will be assessed by visually inspecting the distribution of the funnel plot when there will be at least 10 studies per analysis, ${ }^{41}$ by using Duval and Tweedie's ${ }^{42}$ 'trim and fill' method and by using Egger's ${ }^{43}$ regression test. Comprehensive Meta-Analysis software V.3 $3^{44}$ will be used to conduct all the analyses.

If the necessary data are available, subgroup analyses will be done to verify the association between certain determinants and sleep according to the following variables: population (eg, healthy women vs women with medical conditions, such as cancer), study design (eg, experimental vs observational epidemiological studies) and quality rating (high-rated vs low-rated studies according to the NIH or the EPHPP tool). We are also interested in looking at age differences in sleep. We want to verify whether the data differ according to childbearing age status and also according to pregnancy status since sleep among childbearing age and pregnant women can impact maternal, foetal and child health. ${ }^{14}$ We also want to verify if there are ethnic differences in the determinants of sleep duration and quality among women given that a number of previous reviews have found 'ethnic sleep disparities'. 1213 
Finally, the strength of the evidence will be assessed using the following information, which is derived from the Grading of Recommendations Assessment, Development and Evaluation working group methodology: ${ }^{45}$ (1) quality rating of each study according to the NIH or the EPHPP tool, depending on the study design; (2) magnitude of individual or pooled effect sizes (ie, SMD) according to Cohen's classification, ${ }^{34}$ depending on whether a meta-analysis of the results is possible; (3) heterogeneity between studies using Cochran's $Q^{37}$ and the $\mathrm{I}^{2}$ statistic; $;^{38}$ and (4) publication bias by visually inspecting the distribution of the funnel plot when there will be at least 10 studies per analysis, ${ }^{41}$ by using Duval and Tweedie's ${ }^{42}$ 'trim and fill' method and by using Egger's ${ }^{43}$ regression test.

\section{ETHICS AND DISSEMINATION}

Formal ethical approval is not required as no primary data will be collected. The results will be published in a peer-reviewed journal. This review will provide valuable information on intervention targets for researchers, clinicians and public health stakeholders interested in promoting adequate sleep among women. Since both sleep duration and sleep quality will be investigated, it will be possible to note whether the determinants differ according to the aspect of sleep that is investigated or according to the method used to measure sleep (eg, self-reported vs objective measure). If the findings vary by the aspect of sleep investigated, this could suggest the need to develop different interventions depending on whether the objective is to promote longer and/or improve sleep quality. If the findings vary by the method of assessing sleep, this could suggest the presence of bias or random errors. Investigating simultaneously the individual, social and environmental determinants of women's sleep will allow us to ascertain which determinants contribute the most to adequate sleep in women and provide a more comprehensive picture of factors that affect women's sleep according to ecological models of health behaviour ${ }^{21}$ and socioecological models of determinants of sleep in adults. ${ }^{12}{ }^{13}$ Finally, this review will provide information on whether the determinants of sleep differ according to age (eg, childbearing-age vs older women), pregnancy status (pregnant or not) and ethnicity to inform the development of interventions to promote maternal, foetal and child health in various populations. ${ }^{14}$ This could be useful to tailor interventions to the characteristics of those populations that might be more at risk for short and/or poor sleep. To conclude, the review will identify gaps in knowledge and provide useful information to orient or improve the investigation of determinants of sleep in women and also to design empirically based interventions promoting sleep to favour optimal health and avoid negative health outcomes in women and their children.

Correction notice This paper has been amended since it was published Online First. Owing to a scripting error, some of the publisher names in the references were replaced with 'BMJ Publishing Group'. This only affected the full text version, not the PDF. We have since corrected these errors and the correct publishers have been inserted into the references.

Contributors L-AV-I is the guarantor. L-AV-I drafted the protocol and registered the protocol in PROSPERO. TB reviewed and commented on the protocol in PROSPERO. JPM, DT, TAN and TB all reviewed and commented on this protocol.

Funding L-AV-I is recipient of a fellowship award from the Canadian Institutes of Health Research (CIHR). The funding body had no role in the design of the study, collection, analysis and interpretation of data and in writing the manuscript. This material is based upon work supported by the US Department of Agriculture, Agricultural Research Service under Agreement No. 58-3092-5-001.

Competing interests None declared.

Provenance and peer review Not commissioned; externally peer reviewed.

Open Access This is an Open Access article distributed in accordance with the Creative Commons Attribution Non Commercial (CC BY-NC 4.0) license, which permits others to distribute, remix, adapt, build upon this work non-commercially, and license their derivative works on different terms, provided the original work is properly cited and the use is non-commercial. See: http://creativecommons.org/ licenses/by-nc/4.0/

(c) Article author(s) (or their employer(s) unless otherwise stated in the text of the article) 2017. All rights reserved. No commercial use is permitted unless otherwise expressly granted.

\section{REFERENCES}

1. Grandner MA, Hale L, Moore M, et al. Mortality associated with short sleep duration: the evidence, the possible mechanisms, and the future. Sleep Med Rev 2010;14:191-203.

2. Itani $\mathrm{O}$, Jike $\mathrm{M}$, Watanabe $\mathrm{N}$, et al. Short sleep duration and health outcomes: a systematic review, meta-analysis, and meta-regression. Sleep Med 2017;32:246-56.

3. Lee SW, Ng KY, Chin WK. The impact of sleep amount and sleep quality on glycemic control in type 2 diabetes: a systematic review and meta-analysis. Sleep Med Rev 2017;31:91-101.

4. Fatima Y, Doi SA, Mamun AA. Sleep quality and obesity in young subjects: a meta-analysis. Obes Rev 2016;17:1154-66.

5. Theorell-Haglöw J, Berglund L, Berne C, et al. Both habitual short sleepers and long sleepers are at greater risk of obesity: a population-based 10-year follow-up in women. Sleep Med 2014;15:1204-11.

6. Liu TZ, Xu C, Rota M, et al. Sleep duration and risk of all-cause mortality: a flexible, non-linear, meta-regression of 40 prospective cohort studies. Sleep Med Rev 2017;32:28-36.

7. Nugent CN, Black LI. Sleep duration, quality of sleep, and use of sleep medication, by sex and family type, 2013-2014. NCHS Data Brief 2016;230:1-8.

8. Grandner MA, Jackson NJ, Izci-Balserak B, et al. Social and behavioral determinants of perceived insufficient sleep. Front Neurol 2015;6:112.

9. Gupta S, Wang Z. Predictors of sleep disorders among patients with type 2 diabetes mellitus. Diabetes Metab Syndr 2016;10:213-20.

10. Mehta N, Shafi F, Bhat A. Unique aspects of sleep in women. Mo Med 2015;112:430-4.

11. Roberts $\mathrm{H}$, Hickey M. Managing the menopause: an update. Maturitas 2016;86:53-8.

12. Jackson CL, Redline S, Emmons KM. Sleep as a potential fundamental contributor to disparities in cardiovascular health. Annu Rev Public Health 2015;36:417-40.

13. Grandner MA. Sleep, health, and society. Sleep Med Clin 2017;12:1-22.

14. Ferraro ZM, Chaput JP, Gruslin A, et al. The potential value of sleep hygiene for a healthy pregnancy: a brief review. ISRN Family Med 2014;2014:1-7.

15. Herring SJ, Nelson DB, Pien GW, et al. Objectively measured sleep duration and hyperglycemia in pregnancy. Sleep Med 2014;15:51-5.

16. Qiu C, Enquobahrie D, Frederick IO, et al. Glucose intolerance and gestational diabetes risk in relation to sleep duration and snoring during pregnancy: a pilot study. BMC Womens Health 2010;10:17.

17. Taveras EM, Rifas-Shiman SL, Rich-Edwards JW, et al. Association of maternal short sleep duration with adiposity and cardiometabolic status at 3 years postpartum. Obesity 2011;19:171-8.

18. Amyx M, Xiong $X$, Xie $Y$, et al. Racial/ethnic differences in sleep disorders and reporting of trouble sleeping among women of 
childbearing age in the United States. Matern Child Health $J$ 2017;21:306-14.

19. Bartholomew ELK, Markham CM, Ruiter RAC, et al. Planning health promotion programs: an intervention mapping approach. 4th edition. San Francisco: Jossey-Bass, 2011.

20. Michie S, Atkins L, West R. The behavior change wheel: a guide to designing interventions. Great Britain: Silverback Publishing, 2014.

21. Sallis JF, Owen N, Fisher EB. In: Glanz K, Rimer BK, Viswanath K, Ecological models of health behavior, in Health behavior and health education. Theory, research, and practice. San Francisco, CA: Jossey-Bass, 2008:465-85.

22. Hale L, Emanuele E, James S. Recent updates in the social and environmental determinants of sleep health. Curr Sleep Med Rep 2015;1:212-7.

23. Chang AK, Choi J. Predictors of sleep quality among young adults in Korea: gender differences. Issues Ment Health Nurs 2016;37:918-28.

24. Vitkova M, Rosenberger J, Gdovinova Z, et al. Poor sleep quality in patients with multiple sclerosis: gender differences. Brain Behav 2016;6:e00553.

25. Shamseer L, Moher D, Clarke M, et al. PRISMA-P Group. Preferred reporting items for systematic review and meta-analysis protocols (PRISMA-P) 2015: elaboration and explanation. BMJ 2015;349:g7647.

26. National Sleep Foundation. How much sleep do we really need? 2015. Available from: http://sleepfoundation.org/how-sleep-works/ how-much-sleep-do-we-really-need.

27. Gielen AC, McDonald EM. Using the PRECEDE-PROCEED model to apply health behavior theories. In: Rimer BK, Viswanath K, Glanz K, Health behavior and health education. Theory, research, and practice. San Francisco, CA: Jossey-Bass, 2008:407-33.

28. Carlson MD, Morrison RS. Study design, precision, and validity in observational studies. J Palliat Med 2009;12:77-82.

29. Buysse DJ, Reynolds CF, Monk TH, et al. The Pittsburgh Sleep Quality Index: a new instrument for psychiatric practice and research. Psychiatry Res 1989;28:193-213.

30. Moher D, Liberati A, Tetzlaff J, et al. Preferred reporting items for systematic reviews and meta-analyses: the PRISMA statement. BMJ 2009;339:b2535.
31. National Institute of Health. Quality assessment tool for observational cohort and cross-sectional studies, 2014. (cited 14 Feb 2017).

32. Armijo-Olivo S, Stiles CR, Hagen NA, et al. Assessment of study quality for systematic reviews: a comparison of the Cochrane Collaboration Risk of Bias Tool and the Effective Public Health Practice Project Quality Assessment Tool: methodological research. J Eval Clin Pract 2012;18:12-18.

33. Higgins JPT, Green S. Cochrane Handbook for Systematic Reviews of Interventions. Version 5.0.2 http://handbook.cochrane.org/v5.0.2/. 2011.

34. Cohen J. A power primer. Psychol Bull 1992;112:155-9.

35. Davey J, Turner RM, Clarke MJ, et al. Characteristics of metaanalyses and their component studies in the Cochrane Database of Systematic Reviews: a cross-sectional, descriptive analysis. BMC Med Res Methodol 2011;11:160.

36. Borenstein M, Hedges LV, Higgins JPT, et al. Introduction to metaanalysis. Chippenham: John Wiley \& Sons, 2009.

37. Cochran WG. The $\chi$ test of goodness of fit. Ann Math Stat 1952;23:315-45.

38. Higgins JP, Thompson SG. Quantifying heterogeneity in a metaanalysis. Stat Med 2002;21:1539-58.

39. Huedo-Medina TB, Sánchez-Meca J, Marín-Martínez F, et al. Assessing heterogeneity in meta-analysis: Q statistic or 12 index? Psychol Methods 2006;11:193-206.

40. Higgins JP, Thompson SG, Spiegelhalter DJ. A re-evaluation of random-effects meta-analysis. J $R$ Stat Soc Ser A Stat Soc 2009;172:137-59.

41. Sutton AJ, Duval SJ, Tweedie RL, et al. Empirical assessment of effect of publication bias on meta-analyses. BMJ 2000;320:1574-7.

42. Duval S, Tweedie R. A nonparametric "trim and fill" method of accounting for publication bias in meta-analysis. J Am Stat Assoc 2000;95:89-98.

43. Egger M, Davey Smith G, Schneider M, et al. Bias in meta-analysis detected by a simple, graphical test. BMJ 1997:315:629-34.

44. Borenstein $\mathrm{M}$, et al. Comprehensive meta-analysis version 3. Englewood, NJ: Biostat, 2014.

45. Guyatt GH, Oxman AD, Vist GE, et al. GRADE Working Group. GRADE: an emerging consensus on rating quality of evidence and strength of recommendations. BMJ 2008;336:924-6. 